\title{
A Static Model for Voting on Social Security
}

\author{
Henning Bohn \\ Department of Economics \\ University of California at Santa Barbara \\ Santa Barbara, CA 93106, USA; \\ and CESifo \\ Phone: 1-805-893-4532; Fax: 1-805-893-8830. \\ E-mail: bohn@econ.ucsb.edu \\ http://www.econ.ucsb.edu/ bohn
}

April 2009

JEL Code: H55.

Keywords: Social security, voting model.

\begin{abstract}
This paper examines a static voting model for public pensions. The key premise is that families can internalize the cost and benefits of pay-as-you-go programs. A family realizes a net gain if its members collectively receive more in benefits in the current period than they pay in payroll taxes. Abstracting from differences in income, net benefits are positive if the family's retiree-worker ratio exceeds the national average. If a sufficient fraction of retirees have a suitable number of working-age relatives - not too few and not too many - then a majority of voters belongs to families with above average retiree-worker ratios.
\end{abstract}




\section{Introduction}

Public pensions and other pay-as-you-go financed retirement benefits enjoy strong popular support despite the escalating cost. ${ }^{1}$ This is remarkable from the perspective of voting theory because retiree benefits redistribute resources from a majority of working-age voters to a minority of retirees. Following Aaron (1966) and Browning (1975), a large literature has examined intertemporal models for voting on social security-the notion that contributors may vote in favor because they expect benefits in the future-to explain this puzzle. $^{2}$ Others have appealed to income redistribution and altruism (e.g., Tabellini 2000).

This paper presents an alternative voting model based on intra-family bargaining. The key premise is that families can internalize the cost and benefits of retiree benefit programs. A family realizes a net gain from a pay-as-you-go program if its members collectively receive more in benefits that they pay in payroll taxes. Net benefits are positive if a family's share of retirees exceeds the national average. Preferences over tax rates and benefit levels are single peaked in families' retiree shares.

Retiree benefits are positive in a voting equilibrium if the majority of the population is in families with above average retiree-worker ratios. This condition is plausibly satisfied if most families include a positive, but not too high number of intergenerational linkages. A supporting factor is that if mortality is stochastic, only the survivors vote. For any given structure of family linkages, families with surviving retirees have more members than families with higher mortality. ${ }^{3}$ The conditions for majority support are likely violated, however, in societies with substantial heterogeneity over family linkages, e.g., if most families are have either many children or no children.

\footnotetext{
${ }^{1}$ In the following, I use the term social security to represent a generic pay-as-you-program. The model applies to pensions and to other benefit programs, such as retiree health care.

2 A challenge in dynamic models is to establish a link between current voting and future benefits. The early literature ignored repeated voting. More recently, Cooley and Soarez (1999) have modeled repeated voting with trigger strategy arguments to tie current votes to future benefits. Applications include my own work (Bohn 1999, 2005) and Galasso (2006).

3 With survival uncertainty, retiree benefits can be interpreted as mutual insurance against uncertainty about a needy relative's longevity. A difficulty with this insurance is that ex post, voters whose relatives have died early have an incentive renege. The conditions for majority support for social security can conditions may be viewed as conditions for the political sustainability of survival insurance.
} 
As a technical device to obtain interior solutions for tax rates, I assume payroll taxes distort labor supply. (This avoids extreme shifts between zero taxes and confiscatory taxes in response to small changes in population structure.) Preferences over payroll taxes are then single-peaked as function of families' retiree shares, or equivalently, retiree-worker ratios. In the voting equilibrium, payroll taxes are set so that the marginal excess burden of taxes equals the net financial benefit for the median family.

The model disregards all dynamic voting considerations and assumes working-age voters treat their own future benefits as unrelated to their votes over current benefits. ${ }^{4} \mathrm{I}$ also disregard altruism and income heterogeneity. This is for clarity, to limit the scope of the paper, and to avoid any bias for social security due to altruistic and redistribution features. One may suspect that the remarkable popularity of public retirement and old-age health programs around the world is based on multiple factors. This may include dynamic arguments, altruism, and redistribution across heterogeneous incomes. While it would be straightforward to combine such arguments with the mechanism proposed here, the resulting "combination” models would be unwieldy and they would obscure the conditions under which family bargaining per se is supportive of social security. That is, I view the family bargaining mechanism as complementary with other arguments for social security. ${ }^{5}$

Breyer and v.d. Schulenburg (1987) were the first to propose families as relevant decision units for voting on social security. In contrast to this paper, Breyer and v.d. Schulenburg treat a vote on social security as a once-and-for-all decision and they follow the dynamic literature in assuming that voters base their decisions on a present value of current and expected future taxes and benefits. They also make specific assumptions about family structure, namely that voting-age individuals care about the net taxes of their direct descendents (children, grandchildren, etc.), assuming asexual reproduction (each child having a single parent).

\footnotetext{
4 This is not to deny the relevance of promised future benefits in practice, but to meet the challenge of constructing voter support in a static model - a model that allows repeated voting without requiring trigger strategy arguments. Put differently, I set aside the intertemporal linkages that the dynamic literature works so hard to establish and instead take seriously the simple, non-trigger strategy solution that always exists in trigger strategy models.

${ }^{5}$ Understanding all the reasons why voters support social security is important in part because reform efforts are likely to fail unless they attract majority support. The history of failed reforms in Europe and in the U.S. suggests a need for better understanding.
} 
The paper is organized as follows. Section 2 reviews relevant demographic facts. Section 3 presents the model. Section 4 examines voting behavior and presents the main propositions. Section 5 provides numerical examples. Section 6 concludes.

\section{The Demographics of Families}

The share of retirees in the voting population is around 20\% in the U.S. and in the $25-35 \%$ range in continental Europe. Thus retirees are a strong minority, but far from a majority even in European countries with below-replacement fertility.

The effective retirement age is about 63 in the U.S. and around 60 in Europe. Life expectancy at age 60 is about 20 years-more for females and somewhat less for males. This is well over half of the average time span between generations. Using U.S. life tables, one also finds that for a child of 25year old parents, with about $60 \%$ probability at least one of the parents reaches age 85 . The probability rises to $65 \%$ conditional on both reaching age 60 . This means that a large majority of working-age voters has living parents. For older workers, these parents are likely retired. For children and younger workers, the same argument suggests that a majority will have retired grandparents or grandgrandparents. Thus a large majority of the population has close relatives who benefit from pay-as-yougo public pensions and other retiree benefits (e.g. health care).

Most children are born to mothers age 20-35, with a peak around age 25-30. Hence retirees at, say, age 80 will likely have children in the age range 50-55 and grandchildren in the age range 20-30. This suggests that the voting population encompasses more than two generations and would not captured adequately by modeling a nuclear family. ${ }^{6}$ For this reason, I treat a family as an abstract collection of voters, i.e., without trying to identify specific kinship relations as necessarily more or less relevant than others.

My basic premise is that family linkages may matter for voting. Importantly, the argument does not require altruism towards retired relatives, but merely an ability to align members' voting incentives. For example, a college student with retired grandparents that are supported by his parents

\footnotetext{
6 The longer time spent in working age than in retirement also explains why retirees remain a minority even in countries with fertility below replacement.
} 
might vote against cuts in public pensions because such cuts might trigger a shift in parental support from the student to the grandparents, or perhaps reduced gifts and bequests. In this context, gifts, bequests, and other intra-family payments are not necessarily evidence of altruism, but may reflect Coase-type "side payments" designed to ensure cooperation in voting.

\section{The Model}

Consider an economy with finitely lived individuals. Time is indexed by time $t$, and age is indexed by a. Both are treated as discrete. Individuals born at time t form cohort t. They are eligible to vote age $\mathrm{a}=\mathrm{V} \geq 0$, they become eligible for retirement benefits at age $\mathrm{a}=\mathrm{R}$, where $\mathrm{R}>\mathrm{V}$, and they live up to a terminal age $a=A \geq R$. The labor force consists all non-retired voters, the age range $a \in[V, R-1]$. Let $\pi_{a}$ be the survival function; that is, generation-t individuals survive to (at least) age a with probability $\pi_{\mathrm{a}}$, where $\pi_{\mathrm{a}}>0$ for $\mathrm{a} \leq \mathrm{A}$, declining in $\mathrm{a}$, and $\pi_{\mathrm{A}+1}=0$. Assuming children neither vote nor work, they can be disregarded (their consumption subsumed under their parents consumption); hence $V=0$ is without loss of generality. Then cohort $\mathrm{t}$ is economically active in $[\mathrm{t}, \mathrm{t}+\mathrm{A}]$; and at time $\mathrm{t}$, the living cohorts are those born in [t, t-A].

The economy is deterministic except for stochastic mortality. Labor has an exogenous marginal product of labor $\mathrm{w}$ and savings earn an exogenous real interest $\mathrm{r}$. (Time variation could be added but would just clutter the notation.) The common wage rate serves to emphasize that the model does not rely on intra-cohort redistribution or on a particular income distribution. At age $\mathrm{a}_{0}$, individuals of generation $\mathrm{t}$ have preferences

$$
U_{t}^{a_{0}}=\sum_{a=a_{0}}^{A} \pi_{a} \beta^{a-a_{0}} \bar{u}\left(c_{t-a_{0}+a}^{a}, \lambda_{t-a_{0}+a}^{a}\right)
$$

over consumption $c_{t-a_{0}+a}^{a}$ and leisure $\lambda_{t-a_{0}+a}^{a}$. I abstract from altruism to emphasize that altruism is not essential for the proposed model of social security.

Retiree benefits are structured to pose a one-dimensional voting problem: The retirement age $\mathrm{R}$ is exogenous. Individual at ages $\mathrm{a}<\mathrm{R}$ (workers) pay proportional payroll taxes at rate $\tau_{\tau}$. Retirees (ages $\mathrm{a} \geq \mathrm{R}$ ) receive transfers $\mathrm{T}_{\mathrm{t}}$. Labor supply $l_{t}^{a}=1-\lambda_{t}^{a}$ is endogenous for workers $(\mathrm{a}<\mathrm{R}) ; l_{t}^{a}=0$ is imposed exogenously for ages $\mathrm{a} \geq \mathrm{R}$. Workers’ optimality conditions for labor and leisure are 


$$
\left.\frac{\bar{u}_{\lambda}\left(c_{t}^{a}, 1-l_{t}^{a}\right)}{\bar{u}_{c}\left(c_{t}^{a}, 1-l_{t}^{a}\right)}\right)=w\left(1-\tau_{t}\right) \forall a<R .
$$

To simplify, assume $\mathrm{u}$ has the form $\bar{u}\left(c_{t}^{a}, \lambda_{t}^{a}\right)=u\left(c_{t}^{a}+\phi\left(\lambda_{t}^{a}\right)\right)$, where $\mathrm{u}$ and $\phi$ are increasing and concave. Then labor supply for all $\mathrm{a}<\mathrm{R}$ is characterized by $\phi^{\prime}\left(\lambda_{t}^{a}\right)=w\left(1-\tau_{t}\right)$ and can be written as a declining function of the current tax rate, $l_{t}^{a}=L\left(\tau_{t}\right)$. Assume $\phi$ has a shape such that tax revenues $\tau L(\tau)$ are strictly increasing and strictly concave in $\tau$ up to an interior maximum at some value $\tau^{\max }<1$. (That is, I assume Harberger triangle and Laffer curve intuition applies. This simple treatment of leisure suffices because the tax distortions only serve as device to avoid extreme solutions to the voting problem.) Then $\tau^{\max }$ satisfies $L\left(\tau^{\max }\right)-\tau L^{\prime}\left(\tau^{\max }\right)=0$; and $L(\tau)-\tau L^{\prime}(\tau)$ is positive and strictly decreasing $\tau$ in for $\tau<\tau^{\max }$.

Let $N_{t}^{a}$ denote the number of individuals of age a living in period t. Then

$$
\bar{N}_{t}^{W}=\sum_{a=0}^{R-1} N_{t}^{a}, \bar{N}_{t}^{R}=\sum_{a=R}^{A} N_{t}^{a} \text {, and } L_{t}=\sum_{a=0}^{R-1} \frac{N_{t}^{a}}{N_{t}^{W}} l_{t}^{a}=L\left(\tau_{t}\right)
$$

are the working-age population, the retired population, and the per-worker labor supply, respectively. are the working-age population and the retired population, respectively. Let denote per worker labor supply at time t. ${ }^{7}$

Assuming taxes and transfers are administered pay-as-you-go, the government's budget constraint is $T_{t} \bar{N}_{t}^{R}=\tau_{t} \bar{N}_{t}^{W} w L\left(\tau_{t}\right)$. Every period, the tax rate $\tau_{t}$ is determined by voting decisions, assuming voters understand that transfers $T_{t}=\tau_{t} L\left(\tau_{t}\right) w \cdot \bar{N}_{t}^{W} / \bar{N}_{t}^{R}$ depend on the tax rate $\tau_{t}$ and on the aggregate worker-retiree ratio.

Retiree preferences over $\tau_{\mathrm{t}}$ are strictly increasing in $\tau_{\mathrm{t}}$ whereas worker preferences are strictly decreasing in $\tau_{\mathrm{t}}$, i.e., both are single peaked. If there are more workers than retirees, $\bar{N}_{t}^{W}>\bar{N}_{t}^{R}$, the unique equilibrium with individualistic voting would be $\tau_{\mathrm{t}}=0 .{ }^{8}$ This insight generates the basic puzzle of social security in a democracy: With a majority of voters in working-age, how can one explain the existence of social security?

\footnotetext{
7 If $\varphi_{a}$ denotes the fraction of age-a individuals having a child (fertility), the population dynamics can be described by $N_{t+1}^{a+1}=N_{t}^{a} \cdot \pi_{a+1} / \pi_{a} \forall a<A$ and $N_{t+1}^{0}=\sum_{a=0}^{A} \varphi_{a} N_{t}^{a}$. The dynamics are inessential, however.

${ }^{8}$ In the empirically unrealistic case $\bar{N}_{t}^{W}<\bar{N}_{t}^{R}$, one would obtain $\tau_{\mathrm{t}}=\tau^{\max }$.
} 
The economic literature on social security has tried to resolve the puzzle in various directions, notably by considering dynamic/intertemporal arguments (reputation, generational contracts, trigger strategies), altruism, linkages between intra- and inter-generational redistribution, public choice arguments (the old having more time to lobby), or combinations thereof. For clarity, none of these assumptions is invoked here.

\section{Family Preferences}

The defining feature of a family for the purposes of this paper is the ability to internalize the cost and benefits of government programs that accrue to its members. That is, a family is any collection of individuals with the ability to coordinate its actions in a way that attains the Pareto frontier of its members' utilities.

Families may include direct child-parent-grandparent linkages, higher-order kinship relations (e.g. nephews, uncles), or linkages by marriage. I do not apply specific labels to relationships to avoid ad hoc assumptions about which linkages matter and to keep the notation simple.

Let the economy's population be partitioned into families and consider the Pareto problem of a generic family with $\mathrm{n}_{\mathrm{t}}^{\mathrm{a}}$ members of age a and $n_{t}=\sum_{a=0}^{A} n_{t}^{a}$ total members. Pareto-optimal family allocations can be obtain by maximizing a weighted average of member utilities,

$$
W=\sum_{a=0}^{A} \omega_{a} n_{t}^{a} U_{t}^{a},
$$

for some collection of weights $\omega_{\mathrm{a}}>0$. With or without intertemporal commitments, the period-t decision problem reduces to maximizing

$$
W_{t} \equiv \sum_{a=0}^{A} \omega_{a} n_{t}^{a} u\left(c_{t}^{a}+\phi\left(\lambda_{t}^{a}\right)\right)
$$

subject to $\quad \sum_{a=0}^{A} n_{t}^{a} C_{t}^{a}+S_{t}=\sum_{a=0}^{R-1} n_{t}^{a} \cdot w\left(1-\tau_{t}\right)\left(1-\lambda_{t}^{a}\right)+\sum_{a=R}^{A} n_{t}^{a} T_{t}$,

for any given level of family savings $S_{t}$. Because problem is static, time subscripts are dropped in the following.

While the resulting intra-family allocation of consumption depends on the welfare weights, the conclusions about voting do not, nor do they depend on family wealth or on decisions about savings. 
To see this, differentiate $\mathrm{W}$ with respect to $\tau$ (noting that effects on leisure and on savings cancel out by the envelope theorem) to obtain

$$
\frac{\partial W}{\partial \tau}=\Phi \cdot\left[\sum_{a=R}^{A} n_{t}^{a} \frac{\partial T}{\partial \tau}-\sum_{a=0}^{R-1} n_{t}^{a} w\left(1-\lambda_{t}^{a}\right)\right] .
$$

where $\Phi>0$ is the Lagrange multiplier on the period-t resource constraint.

Note that $1-\lambda_{t}^{a}=L(\tau)$ and $\frac{\partial T}{\partial \tau}=\left[L(\tau)+\tau L^{\prime}(\tau)\right] w \frac{N_{t}^{W}}{N_{t}^{R+}}=\left[L(\tau)+\tau L^{\prime}(\tau)\right] w \frac{1-\bar{\eta}}{\bar{\eta}}$, where $\bar{\eta}=N_{t}^{R+} /\left(N_{t}^{R+}+N_{t}^{W}\right) \in(0,1)$ denotes the aggregate population share of retirees. Similarly, let $\eta=\sum_{a=R}^{A} n_{t}^{a} / n_{t} \in[0,1]$ denote the family's own share of retirees. Then a family's optimality condition for taxes can be written as

$$
\frac{\partial W}{\partial \tau}=\Phi w(1-\bar{\eta}) n_{t} \cdot\left[\left[L(\tau)+\tau L^{\prime}(\tau)\right] \frac{\eta}{\bar{\eta}}-L(\tau) \frac{1-\eta}{1-\bar{\eta}}\right]=0,
$$

where the second equality must hold at interior solutions. (For $\eta<1, \eta /(1-\eta)$ can be interpreted as the retiree-worker ratio, a more commonly used variable. I use the retiree-share for the analysis because the retiree share is finite and bounded even for families without workers, for which there is no finite retiree-worker ratio.)

Noting that $\partial W / \partial \tau$ is increasing in $\eta$, one finds:

Proposition 1: Family preferences over payroll taxes are a single-peaked function of the retiree share. The preferred values satisfy $\tau^{*}(\eta)=0$ for families with $\eta \leq \bar{\eta} ; \tau^{*}(\eta) \in\left(0, \tau^{\max }\right)$ for $\bar{\eta}<\eta<1$ with $\tau^{*}(\eta)$ strictly increasing in $\eta$; and $\tau^{*}(\eta)=\tau^{\max }$ for $\eta=1$.

Proof: From (1), an interior solution for the preferred tax rate $\tau^{*}$ must satisfy $\left[L(\tau)+\tau L^{\prime}(\tau)\right] \frac{\eta}{\bar{\eta}}=L(\tau) \frac{1-\eta}{1-\bar{\eta}}$. For $\eta=1, \tau^{*}$ this reduces to $L(\tau)+\tau L^{\prime}(\tau)=0$, which means $\tau^{*}=\tau^{\max }$. For $\eta=\bar{\eta}<1$, the condition reduces to $L^{\prime}(\tau)=0$, which implies $\tau^{*}=0$. Because $L^{\prime}(\tau)<0$ for all $\tau>0, \tau^{*}=0$ applies as corner solution for all $\eta<\bar{\eta}$. For $\bar{\eta}<\eta<1$, $\left[1+\tau \frac{L^{\prime}(\tau)}{L(\tau)}\right]=\frac{\bar{\eta} /(1-\bar{\eta})}{\eta /(1-\eta)} \in(0,1)$, where the r.h.s. is decreases strictly in $\eta$. Because the l.h.s. is strictly decreasing in $\tau$, the preferred value is unique and increasing in $\eta$. For all $\eta$, preferences are single peaked because $\partial W / \partial \tau$ changes sign only once, from positive to negative at $\tau^{*}$. QED. 
Proposition 1 implies that family preferences are also single peaked in the family's retiree-worker ratio.

The economic intuition why only the retiree share matters is based on Coasian reasoning. In countries where retirees are not entitled to family support, retirees may have to make side-payments to induce working-age relatives to endorse positive payroll taxes. In countries where children are obliged to support needy parents (by law or perhaps by custom), no side payments may be needed, or payments may go from working-age to retired members. ${ }^{9}$

In the limiting case of (almost) inelastic labor supply, $L^{\prime}(\tau) \rightarrow 0$ would imply $\tau^{\max } \rightarrow 1$ and $\tau^{*}(\eta) \rightarrow 1$ for all $\eta>\bar{\eta}$. That is, preferred tax rates would diverge to the extremes of the unit interval. The assumption of quasi-linear preferences over leisure is meant as a smoothing device, to rule out such extreme solutions and to ensure continuous preferences over the size of the social security system.

To define a voting equilibrium, let individuals be sorted by the retiree shares of the families they belong to. Let $F(\eta)$ denote the resulting cumulative distribution function of voters. That is, in a multi-person families, the family's $\eta$ value is counted as many times as the family has members. Single, unattached workers and retirees are interpreted as families of size one with retiree shares $\eta=0$ and $\eta=1$, respectively. Let $\eta_{\text {med }}=F^{-1}(1 / 2)$ denote the retiree share of the median voter's family. Then:

\section{Proposition 2:}

(a) A unique voting equilibrium exists and is given by $\tau^{*}=\tau *\left(\eta_{\text {med }}\right)$.

(b) The equilibrium payroll tax rate $\tau *$ is strictly positive if and only if $\eta_{\text {med }}>\bar{\eta}$.

Proof: Follows from Prop.1 and the definition of $\eta_{\text {med. }}$ QED.

\footnotetext{
${ }^{9}$ Note that $\partial W / \partial \tau$ does not depend on welfare weights. The irrelevance of welfare weights also implies that the sign of $\partial W / \partial \tau$ would remain unchanged if individuals had preferences that put positive weight on their relatives' $U_{t}^{a_{0}}$ functions. It would be straightforward to include this type of altruism formally, but requiring tedious notation and regularity conditions. Altruism would influence the amount and direction of side-payments.
} 
The key condition for the existence of social security is that the majority of the population lives in families with retiree share greater than the aggregate retiree share; or equivalently, that the majority lives in families with retiree-worker ratios greater than the aggregate retiree-worker ratio.

\section{Examples}

A natural next question is which assumptions about demographics are likely to satisfy the condition $\eta_{\text {med }}>\bar{\eta}$. A study of the empirical distribution of family sizes is beyond the scope of this paper, and it may not be persuasive anyway because one would have to make ad hoc assumptions about the strengths of various linkages. Hence I focus on examples that illustrate the basic forces driving the voting outcomes.

\section{Example \#1 [Two point distribution]}

Suppose a fraction $\mathrm{f}$ of working-age individuals forms lasting bonds with $\mathrm{m}>0$ family members who will be voting age when the person is in retirement—called intergenerational bonds in the following. Natural examples would be bonds with children—some or all—but the bonds could be with other younger relatives like grandchildren, nieces or nephews, or in-laws. Assume a fraction $\pi_{R} \in(0,1]$ of the working-age population lives to the maximum age $\mathrm{A}$, and fraction 1- $\pi_{\mathrm{R}}$ dies at the retirement age R. Let $\hat{\eta}=\bar{\eta} / \pi_{R}$ be the hypothetical retired population if all retirees had survived, expressed as share of actual population. Assume retirees are not linked to each other and that each working-age voter is linked to at most one retiree. Then $\mathrm{f}$ and $\mathrm{m}$ are bounded by $f m \leq(1-\bar{\eta}) / \hat{\eta}$, or $f m \leq \pi_{R}(1-\bar{\eta}) / \bar{\eta}$. Also, assume $\bar{\eta}<1 / 2$ so retirees are a minority.

The example economy has three types of families. Retirees without intergenerational bonds form single-member families; they account for share $\pi_{R} \hat{\eta}(1-f)=\bar{\eta}(1-f)$ of the population. A fraction $f$ of retirees lives in families consisting of the retiree plus $m$ workers. Such families have retiree share $\eta=1 /(1+m)>0$ and their population share is $\pi_{R}(1+m) \hat{\eta} f=(1+m) \bar{\eta} f$. Finally, families have workers and no retirees, either because the workers never bonded with a retiree (population share $1-\bar{\eta}-f m \hat{\eta}$ ) or because the retired member has died (population share $\left.\left(1-\pi_{R}\right) f m \hat{\eta}\right)$. The first type 
prefers $\tau^{*}(1)=\tau^{\max }$ but is a minority. The second type prefers $\tau^{*}(\eta)>0$ if and only if $\eta=1 /(1+m)>\bar{\eta}$. The third type prefers $\tau^{*}(0)=0$ and constitutes a majority if and only if

$$
F(0)=1-\bar{\eta}-f m \hat{\eta}+\left(1-\pi_{R}\right) f m \hat{\eta}=1-\bar{\eta}-f m \bar{\eta}>1 / 2 .{ }^{10}
$$

With this population structure, social security taxes are positive if and only if

(A) the median voter's family is a family of retirees with workers: $1-\bar{\eta}-f m \bar{\eta}<1 / 2$; and

(B) this type of family prefers positive taxes: $1 /(1+m)>\bar{\eta}$.

Condition (A) imposes a lower bound on the number of workers linked to retirees, $f m>(1 / 2-\bar{\eta}) / \bar{\eta}$. Because $f m \leq \pi_{R}(1-\bar{\eta}) / \bar{\eta}$, (A) also requires a sufficiently high survival rate, namely $\pi_{R}>(1 / 2-\bar{\eta}) /(1-\bar{\eta})$. Condition (B) imposes an upper bound on the number of workers in each worker-retiree family, $m<(1-\bar{\eta}) / \bar{\eta}$. For $\bar{\eta}<1 / 2$, the interval $[(1 / 2-\bar{\eta}) / \bar{\eta},(1-\bar{\eta}) / \bar{\eta}]$ has a length exceeding one and therefore includes one or more integers $\mathrm{m}$. For integers $\mathrm{m}$ in this interval, (A) holds if $\pi_{\mathrm{R}}$ is sufficiently close to one, and (B) holds if $\mathrm{f}$ is sufficiently close to one. Thus, voting solutions with positive taxes do exist.

Quantitatively, retiree shares in developed countries are in the 0.2 to 0.35 range. Consider $\bar{\eta}=0.3$ as specific example. One obtains $(1-\bar{\eta}) / \bar{\eta}=7 / 3$ and $(1 / 2-\bar{\eta}) / \bar{\eta}=2 / 3$. Hence conditions (A) and (B) are satisfied for $m=1$ with $f>2 / 3$ and for $m=2$ with $f>1 / 3$. That is, family linkages support social security if there are a sufficient number of "small” families-either at least 2/3 of retirees in families with one working-age member or at least $1 / 3$ of retirees in families with exactly two workingage members. Social security does not have majority support if either too few retirees have intergenerational bonds, or if retirees with intergenerational bonds are linked to too many workers.

Table 1 displays, for a range of retiree shares, combinations of family sizes (m), minimal survival rates $\left(\pi_{R}\right)$, and minimal frequency of family bonds (f) that support social security. For retireeshares in the over-1/4 to near-1/2 range, a significant fraction of one-worker-one-retiree linkages suffices, or a smaller number of multiple linkages. When retiree shares are lower, social security requires a larger number of family linkages (higher $\mathrm{m}$ ) to bind a sufficient number of workers into

\footnotetext{
10 Throughout, I disregard the case of tied, 50:50 voters, because they would distract from substantive insights and because such cases are non-generic in the parameter spaces considered.
} 
families with retirees. In addition, the number of links must not be too high because a family with too many workers would have a retiree share below the national average and prefer zero taxes.

Note that a survival probability of less than $1 / 2$ suffices in all cases. Because the survivor him/herself can vote, families with retirees can have a majority of votes even if retirees have died in a majority of families. If social security is interpreted as insurance against the cost of supporting family members who survive into retirement, Table 1 can be interpreted as providing conditions under which families with early deaths cannot renege on their insurance payments.

Overall, Table 1 suggests that family support for social security requires a balance between retiree shares and family sizes. Societies with high fertility rates tend to have large families and low retiree shares. In such societies, static support for social security requires that most retiree are linked to a substantial number of working-age family members. In societies with low fertility, where families tend to be small and retiree shares high, support for social security requires appropriately smaller number and/or frequency of family bonds. Prior to the demographic transition, survival rates into retirement were probably too low to support social security even with large families. Thus the model is consistent with the introduction of social security during the demographic transition, when retiree shares and survival rates increased while family size decreased.

Note that the equilibrium tax rate $\tau *\left(\frac{1}{1+m}\right)$ is increasing as $m$ decreases. Hence a social security system backed by size $m=1$ families has a higher tax rate than a social security system supported by size $m>>1$ families. This suggests that the historical growth of social security may be related to the historical trend towards smaller families and not (only) to the aging process per se.

\section{Example \#2 [General distribution]}

The assumption in Example 1 of a common family size for all worker-and-retiree families is clearly restrictive. Consider now the same setting, but with a distribution over the number of family bonds. Let $\pi_{\mathrm{m}}$ be the fraction of retirees with m links, with m ranging from zero to some maximum value M. Let $\bar{m}$ be the average number of family bonds per retiree. Note that, analogous to Example 1, 
$\bar{m} \leq(1-\bar{\eta}) / \hat{\eta}=\pi_{R}(1-\bar{\eta}) / \bar{\eta}$, and that social security requires less than $50 \%$ population share of workers-only families, $1-\bar{\eta}-\bar{m} \cdot \bar{\eta}<1 / 2$.

Among families with retirees, those with $\eta=1 /(1+m)>\bar{\eta}$ (which always includes $m=1$ ) will prefer $\tau^{*}(\eta)>0$. Families with $1 /(1+m)<\bar{\eta}$ prefer $\tau^{*}(\eta)=0$. Support for social security is thus concentrated among relatively small families with a retiree, whereas larger families would be better off supporting their retiree privately. Let $\breve{m}=\lfloor(1-\bar{\eta}) / \bar{\eta}\rfloor$ denote the highest m-value for which families support social security. Then a voting equilibrium has positive social security taxes if and only if 11

$$
\Sigma_{0}=\sum_{m=0}^{\breve{m}} \pi_{m}(1+m) \cdot \bar{\eta}>1 / 2
$$

Condition (2) ensures that families with retiree and up to $\breve{m}$ workers constitute a majority. Unless the jump from $(1-\bar{\eta}) / \bar{\eta}$ to the next lower integer exceeds $(1-\bar{\eta}) / \bar{\eta}-\bar{m}$, the $\bar{m}$ exceeds $\bar{m}$. If (2) is satisfied, let $m^{*} \in\{1, \ldots, \breve{m}\}$ be the lowest integer for which $\sum_{m=0}^{m^{*}} \pi_{m}(1+m) \cdot \bar{\eta}>1 / 2$. Then the equilibrium tax rate is $\tau^{*}\left(\frac{1}{1+m^{*}}\right)$ and it is decreasing in $m^{*}$.

For a quantitative illustration, consider again $\bar{\eta}=0.3$, which implies $\breve{m}=2$. Condition (2) then specializes to $\Sigma_{0}=0.3 \cdot \pi_{0}+0.6 \cdot \pi_{1}+0.9 \cdot \pi_{2}>0.5$. Sufficient conditions are $\pi_{2}>5 / 9$, or $\pi_{1}>5 / 6$, or a linear combination where any gap between $\pi_{2}$ and 5/9 is covered by sufficient $\pi_{1}$ and $\pi_{0}$ values. The median voter's family size is $m^{*}=1$ if $0.3 \cdot \pi_{0}+0.6 \cdot \pi_{1}>0.5$, which means that $\pi_{1}$ plus half of $\pi_{0}$ must exceed 5/6; otherwise $m^{*}=2$.

Note that families with $m>\breve{m}$ vote against social security. The model is thus consistent with the absence of social security systems in societies with many large families. The least supportive environment for social security would be a combination of disconnected retirees $(m=0)$ and families of size $m=M=\breve{m}+1$. This scenario would maximize the number of retirees in families that vote for no taxes. Only the fraction $\pi_{0}$ of retirees would support social security.

Retiree-only families do help to satisfy condition (2), but because the weight on $\pi_{0}$ is less than $1 / 2$, their support is insufficient. Families ties between retirees and a small but positive number of workers are therefore central to generating voting support for social security.

\footnotetext{
11 To cover cases with $\breve{m}>M$, formally define $\pi_{\mathrm{m}}=0$ for $\mathrm{m}>\mathrm{M}$.
} 
Examples 1-2 do not allow for family bonds between retirees. Given the institution of marriage, this is restrictive. Turning to family structures with multiple retirees, key questions are how the number of working-age members grows with the number of retired members, and to what extent the death of a retiree breaks linkages between surviving retirees and working-age members. In some cases, one may suspect multiple bonds that survive the death of one retiree (e.g., married retirees with common children), whereas in other cases each retiree may contribute a separate set of intergenerational bonds. If marriage correlates with children or otherwise helps to coordinate voting among a larger number of members, two-retiree families may even have more than twice the number of family bonds than one-retiree families.

\section{Example \#3 [Multiple retirees]}

Consider the same setting as in Example 2, but allow for families with two retirees. (A larger number could be modeled along the same lines, but the case of one- and two-retiree families suffices to illustrate the new issues.) Suppose a fraction $\mu$ of prospective retirees establishes, during working-age, a family bond with a same-age partner—labeled marriage for brevity—plus up to M bonds to younger members who will be working-age when the senior members are retired, i.e., intergenerational bonds. A fraction $1-\mu$ is never married and forms $m$ intergenerational bonds with probability $\pi_{\mathrm{m}}$, as in Example 2. Assume survival into retirement is independent of a partner's survival. Then a fraction $\mu \cdot \pi_{R}^{2} \cdot \hat{\eta}=\mu \cdot \pi_{R} \cdot \bar{\eta}$ of the population are married retirees, a fraction $\mu \cdot\left(1-\pi_{R}\right) \cdot \bar{\eta}$ are widowed retirees, and $(1-\mu) \cdot \bar{\eta}$ are never-married retirees.

Social security is again supported by families with retirees and with not too many working-age members. Let $\breve{m}=\lfloor(1-\bar{\eta}) / \bar{\eta}\rfloor$ and $\breve{m}_{2}=\lfloor 2(1-\bar{\eta}) / \bar{\eta}\rfloor$ denote the highest m-values for which oneand two-retiree families support social security, respectively. If $\breve{m}_{2}$ is even, $\breve{m}_{2}=2 \breve{m}$, otherwise $\breve{m}_{2}=2 \breve{m}+1$. A voting equilibrium has positive social security taxes if single-retiree families with $m \leq \breve{m}$ plus two-retiree families with $m \leq \breve{m}_{2}$ constitute a majority. 
As benchmark, suppose intergenerational bonds in two-retiree families are additive in the sense that each partner contributes bonds according to the distribution $\pi_{\mathrm{m}}$ and that these links are broken when the contributing partner dies. Then intergenerational bonds in two-retiree families are distributed $\pi_{2, m}=\sum_{i=\min (m, M)}^{\max (m, M)} \pi_{i} \pi_{m-i}$ for $m \leq 2 M$, the distribution of the sum. The condition for positive social security taxes is

$$
\Sigma_{1}=\sum_{m=0}^{\breve{m}} \pi_{m}(1+m) \cdot\left[1-\mu+\mu\left(1-\pi_{R}\right)\right] \bar{\eta}+\sum_{m=0}^{\breve{m}_{2}} \pi_{2, m}(1+m / 2) \cdot \mu \pi_{R} \bar{\eta}>1 / 2 .
$$

Compared to Example 2, one finds

$$
\Sigma_{1}=\Sigma_{0}+\left\{\sum_{m=0}^{\breve{m}_{2}} \pi_{2, m}(1+m / 2)-\sum_{m=0}^{\breve{m}} \pi_{m}(1+m)\right\} \cdot \mu \pi_{R} \bar{\eta} .
$$

Note that children per retiree in two-retiree families have a distribution with the same mean $\bar{m}$ as one-retiree families, but with only half the variance. Recall that the cutoff $\breve{m}$ tends to be above the mean. This suggests that the fraction of two-retiree families with $m \leq \breve{m}_{2}$ will exceed the fraction of one-retiree families with $m \leq \breve{m}$, and thus marriage increases the support for social security. This intuition does not always apply, however, because voting support is lost whenever a retiree with $m \leq \breve{m}$ is paired with a retiree who contributes more than $\breve{m}_{2}-m$ additional intergenerational bonds.

For a quantitative illustration, suppose $\bar{\eta}=0.3$, which implies $\breve{m}=2$ and $\breve{m}_{2}=4$. In addition, assume $\mu=\pi_{R}=0.7$ so $49 \%$ of retirees are surviving married and $21 \%$ widowed. ${ }^{12}$ For the distribution $\pi_{0}=.4$ and $\pi_{1}=\pi_{2}=.30$, one finds $\Sigma_{1}=\Sigma_{0}=0.57$. Marriage does not affect voting outcomes. (One can show that this irrelevance applies in general whenever $\breve{m} \geq M$.) For $\pi_{0}=.35, \pi_{1}=\pi_{2}=.30$, and $\pi_{3}=.05, \Sigma_{1}=0.5675$ exceeds $\Sigma_{0}=0.5555$, so voter support is increased. While the support condition $\mathrm{m} \leq 2$ fails for $5 \%$ of one-retiree families, condition $m \leq 4$ fails for only $3.25 \%$ of two-retiree families. For $\pi_{0}=.35, \pi_{1}=\pi_{2}=.30$, and $\pi_{5}=.05$, in contrast, $\Sigma_{1}=0.5414$ is less than $\Sigma_{0}=0.5555$, so voter support is decreased. This is because families with $m=5$ will voter against social security even with two retirees.

Different assumptions about two-retiree families yield similarly mixed results. For example, suppose marriage does not add intergenerational bonds but creates linkages that survive the other

\footnotetext{
12 Note that because $\mu$ and $\pi_{\mathrm{R}}$ only determine the weight on the bracket expression in (4) and not the sign of $\Sigma_{1}-\Sigma_{0}$, they do not matter for the key question if multiple-retiree families retiree partnerships increases or reduces voter support; also note that $\mu$ and $\pi_{\mathrm{R}}$ enter only through their product. The key issue is thus the distribution of family bonds-the shapes of $\pi_{\mathrm{m}}$ and $\pi_{2 m}$ and their interaction.
} 
retiree's death. Then the distribution $\pi_{\mathrm{m}}$ applies to all families with retirees, and the condition for positive social security taxes is

$$
\Sigma_{2}=\sum_{m=0}^{\breve{m}} \pi_{m}(1+m) \cdot\left[1-\mu+\mu\left(1-\pi_{R}\right)\right] \bar{\eta}+\sum_{m=0}^{\breve{m}_{2}} \pi_{m}(1+m / 2) \cdot \mu \pi_{R} \bar{\eta}>1 / 2 .
$$

Compared to the previous cases, one finds

$$
\begin{aligned}
\Sigma_{2} & =\Sigma_{0}+\left\{\sum_{m=0}^{\breve{m}_{2}} \pi_{m}(1+m / 2)-\sum_{m=0}^{\breve{m}} \pi_{m}(1+m)\right\} \cdot \mu \pi_{R} \bar{\eta} \\
& =\Sigma_{1}+\left\{\sum_{m=0}^{\breve{m}_{2}}\left(\pi_{m}-\pi_{2, m}\right)(1+m / 2)\right\} \cdot \mu \pi_{R} \bar{\eta}
\end{aligned}
$$

In this case, marriage turns families with $m \in\left[\breve{m}+1, \breve{m}_{2}\right]$ workers into social security supporters. But in families with $m \in[1, \breve{m}]$ workers, where one retiree would have been enough to gain the workers' support, a second retiree may represent a lost opportunity to form a separate family that includes additional workers. The net effect can be positive or negative, depending on how much support is gained for $m \in\left[\breve{m}+1, \breve{m}_{2}\right]$ versus support lost for $m \in[1, \breve{m}] .^{13}$

Overall, Example 3 suggests that multi-retiree families complicate the analysis without necessarily affecting the results in either direction.

Figure 1 plots the cutoff values $\breve{m}$ and $\breve{m}_{2}$ against the retiree share $\bar{\eta}$. Two specific scenarios may be instructive, one for the U.S., the other for continental Europe. First, suppose $\bar{\eta}$ is equal or slightly below 0.2 , the relevant range for the U.S. Then families with one retiree and up to 4 workingage members plus families with two retirees and up to 8 working-age members will support social security. It seems plausible that these conditions cover the vast majority of American families—all but very large extended families. Second, suppose $\bar{\eta}$ is around 0.3 , the relevant range in continental European countries. Then families with one retiree and up to two working-age members, plus families with 2 retirees and up to 4 working-age members, will support social security. Because families with more than two children have become rare in Europe, and because even families with two retirees, two children, and two grandchildren would also satisfy these conditions, the conditions are plausibly satisfied in European countries, too.

\footnotetext{
13 For example, consider again $\pi_{0}=.4, \pi_{1}=\pi_{2}=.30$, and $\pi_{3}=.05$, with the same other assumptions as above. Then $\Sigma_{2}=0.5072$ is less than $\Sigma_{0}$ and $\Sigma_{1}$, but still above $50 \%$.
} 


\section{Conclusions}

The paper provides conditions for pay-as-you-go benefits to retirees to be supported as a voting equilibrium in a static model. The key requirement is that a sufficient fraction of retirees are in families with a positive, but not excessively large number of working-age members. Then the median voter lives in a family with above-average retiree share. Numerical examples suggest that this condition is satisfied in Europe and in the United States.

The paper's focus on static family bargaining is not to dispute the relevance of other voting arguments. It would straightforward, for example, to make labor productivity heterogeneous and to show that a skewed wage distribution would increase voter support for public pensions with progressive benefits. The model is also potentially complementary to dynamic models; many such models rely on trigger strategies that (arbitrarily) select one of many equilibrium paths that could have been supported by alternative beliefs about voting in the future. The static argument for social security provides a natural lower bound for expectations about future benefits.

Overall, the mechanism proposed here helps to explain the remarkable popularity of public retirement and retiree health care programs. This is important because understanding the popularity of such programs is arguably a pre-condition for designing politically feasible reforms. 


\section{References}

Aaron, H.J., 1966. The Social Insurance Paradox, Canadian Journal of Economics and Political Science 32, 371-376.

Bohn, Henning, 1999. Will Social Security and Medicare Remain Viable as the U.S. Population is Aging? Carnegie-Rochester Conference Series on Public Policy 50, 1-53. , 2005. Will Social Security and Medicare Remain Viable as the U.S. Population is Aging? An Update. In: Robin Brooks and Assaf Razin (eds.), The Politics and Finance of Social Security Reform, Cambridge University Press, 44-72.

Breyer, Friedrich and J-Matthias Graf v. d. Schulenburg, 1987. Voting on Social Security: The Family as Decision-Making Unit. Kyklos 40(4), 529-547.

Browning, E.K. (1975). Why the Social Insurance Budget is too large in a Democratic Society. Economic Inquiry 13, 373-388.

Cooley, Thomas, and Jorge Soarez, 1999. A Positive Theory of Social Security Based on Reputation, Journal of Political Economy 107, 135-160.

Galasso, Vincenzo, 2006. The Political Future of Social Security in Aging Societies. MIT Press.

Tabellini, Guido, 2000, A Positive Theory of Social Security. The Scandinavian Journal of Economics 102(3), 523-545. 


\section{Table 1: Conditions for Social Security in Example 1}

\begin{tabular}{|c|c|c|c|c|c|}
\hline \multirow{2}{*}{$\begin{array}{c}\text { Retiree } \\
\text { Share }(\bar{\eta}) \\
\text { Given }\end{array}$} & \multirow{2}{*}{$\begin{array}{l}\text { Survival Prob. } \\
\qquad\left(\pi_{R}\right) \\
\text { Lower bound }\end{array}$} & \multicolumn{3}{|c|}{$\begin{array}{l}\text { Conditions on the number of family links } \\
(\mathrm{m})\end{array}$} & \multirow{2}{*}{$\begin{array}{l}\text { Fraction linked } \\
\text { (f) } \\
\text { Lower bound }\end{array}$} \\
\hline & & $\begin{array}{l}\text { Lower } \\
\text { bound }\end{array}$ & $\begin{array}{l}\text { Upper } \\
\text { bound } \\
\end{array}$ & Integer m & \\
\hline \multirow[t]{5}{*}{0.10} & 0.44 & 4.0 & 9.0 & 5 & 0.80 \\
\hline & & & & 6 & 0.67 \\
\hline & & & & 7 & 0.57 \\
\hline & & & & 8 & 0.50 \\
\hline & & & & 9 & 0.44 \\
\hline \multirow[t]{3}{*}{0.15} & 0.41 & 2.3 & 5.7 & 3 & 0.78 \\
\hline & & & & 4 & 0.58 \\
\hline & & & & 5 & 0.47 \\
\hline \multirow[t]{3}{*}{0.20} & 0.38 & 1.5 & 4.0 & 2 & 0.75 \\
\hline & & & & 3 & 0.50 \\
\hline & & & & 4 & 0.38 \\
\hline \multirow[t]{2}{*}{0.25} & 0.33 & 1.0 & 3.0 & 2 & 0.50 \\
\hline & & & & 3 & 0.33 \\
\hline \multirow[t]{2}{*}{0.30} & 0.29 & 0.7 & 2.3 & 1 & 0.67 \\
\hline & & & & 2 & 0.33 \\
\hline 0.35 & 0.23 & 0.4 & 1.9 & 1 & 0.43 \\
\hline 0.40 & 0.17 & 0.3 & 1.5 & 1 & 0.25 \\
\hline 0.45 & 0.09 & 0.1 & 1.2 & 1 & 0.11 \\
\hline
\end{tabular}


Figure 1: Maximum number of working-age members for families with one and two retirees to support social security

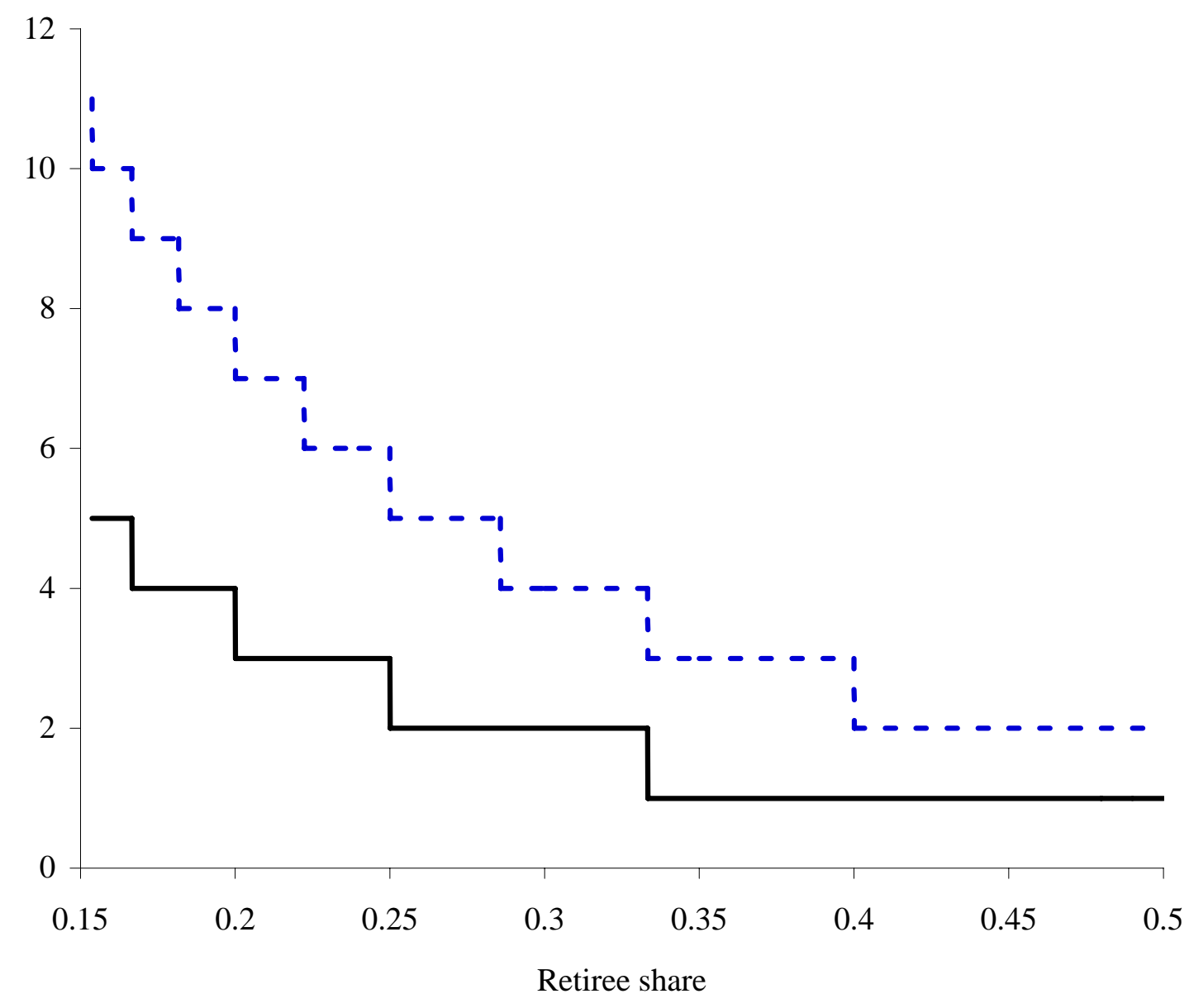

1-retiree families - - - 2-retiree families 